\title{
Betrachtungen zur Durchseuchung und beobachteter Aktivität bei Influenza B
}

\author{
Von H. Uphoff, R. Heckler und B. Schweiger
}

\section{Zusammenfassung}

Anhand der Influenza-Surveillancedaten von $1988 / 89$ bis $1997 / 98$ wurde für Influenza B ein einfaches Modell für das Wechselspiel von Populationsimmunität und Influenzaaktivität entwickelt. Dafür wurde ein Index für die Populationsimmunität aus der Verbreitung der Influenza $B$ in den fünf vorangegangenen Beobachtungsperioden bestimmt. Die Ermittlung der anzunehmenden Abnahme der Bedeutung der Durchseu chung mit der Zeit, aufgrund der Drift und sinkender Antikörpertiter, erfolgte anhand des Modells mit empirischen Methoden. Es konnte eine enge Korrelation zwischen Immunitätsindex und beobachteter Verbreitung der Influenza $B$ innerhalb der sechs aus den Daten berechenbaren Beobachtungsperioden (1992/93 bis 1997/98) hergestellt werden.

\section{Einleitung}

Influenza verursacht fast in jedem Jahr einen deutlichen Anstieg der Morbidität. In Europa wird die erhöhte Influenzaaktivität mit wenigen Ausnahmen im Winterhalbjahr beobachtet. Die Zahl der Erkrankungen ist von Jahr zu Jahr unterschiedlich. In unregelmäßigen $\mathrm{Ab}$ ständen werden auch in interpandemischen Phasen sehr hohe Morbiditäten beobachtet, die enorme Kosten, eine hohe Zahl an schweren Krankheitsverläufen und Komplikationen verursachen sowie zu deutlichen Übersterblichkeiten führen. Bisher ist es - außer bei Shiftsituationen - nicht gelungen, solche "Epidemien" mit bestimmten epidemiologischen, virologischen oder klimatischen Faktoren eng zu korrelieren.

Es wird jedoch angenommen, daß die Immunität der Bevölkerung ein wesentliches Moment der Influenza-Verbreitung darstellt. Als ein wichtiger Faktor für die Populationsimmunität wiederum kann die Durchseuchung in den vergangenen Jahren angesehen werden. In der menschlichen Population spielen die beiden Influenzatypen $A$ und $B$ sowie seit mehr als 20 Jahren die Subtypen der Influenza A, H3N2 und H1N1 eine Rolle. Zwischen Influenza A und B besteht keine Kreuzimmunität, das heißt, eine abgelaufene Erkrankung mit Influenza A schützt nicht vor einer Infektion mit Influenza B und umgekehrt. Die Kreuzimmunitäten zwischen den beiden Subtypen AH3N2 und AH1N1 werden als sehr gering eingestuft. Daher muß die Populationsimmunität gegenüber den Influenzatypen und -subtypen getrennt betrachtet werden.

Die Bedeutung der Durchseuchung vorangegangener Jahre für die Populationsimmunität nimmt mit der Zeit ab. Dabei ist im einzelnen nicht bekannt, welche Rolle die Abnahme der Titer an protektiven Antikörpern nach einer Infektion oder andere zeitabhängige Veränderung der Immunität haben. Bei den Influenzatypen und -subtypen spielt auch die Drift, also die Veränderung der Oberflächenantigene durch Punktmutationen, eine wichtige Rolle, da hierdurch die Immunität, die durch vorangegangene Infektionen erworben wurde, unterlaufen wird. Virologisch wird die Drift mit Hilfe der Feintypisierung (Hämagglutinationsinhibitionstest mit verschiedenen Antiseren) und $\mathrm{der} \mathrm{Se}$ quenzierung des Hämagglutinins erfaßt. Diese Methoden erlauben jedoch keine ausreichende Bewertung der Kreuzreaktionen verschiedener Driftvarianten für die Populationsimmunität.

$\mathrm{Ob}$ und inwieweit weitere Faktoren die Populationsimmunität beeinflussen können, ist im einzelnen nicht bekannt.

Als Datengrundlage für eine modellhafte Untersuchung der oben genannten theoretischen Annahmen kommen Serosurveillance, Morbiditätsbeobachtungen und virologische Untersuchungen in Betracht.

Eine Voraussetzung dafür, Morbiditätsdaten zu nutzen, ist eine hohe Qualität derselben. Die epidemiologischen Beobachtungen in Deutschland haben sich in den vergangenen Jahren deutlich verbessert. Die Daten zur Morbidität werden bei einem aussagefähigen Anteil der Bevölkerung mit Hilfe von primärversorgenden Arztpraxen (Sentinel) erhoben und unterliegen somit einer gerin- gen Selektion nach dem Schweregrad der Erkrankung [1]. Die Zahl und Methoden der virologischen Untersuchungen wurden ebenfalls deutlich verbessert, und die untersuchten Proben stammen überwiegend aus der in die epidemiologischen Beobachtungen eingeschlossenen Bevölkerungsstichprobe [2].

Unter diesen Voraussetzungen erscheint die Annahme gerechtfertigt, daß 1. die mit dem Sentinel beobachtete und der Influenza zuzuordnende Morbidität einer Beobachtungsperiode die Durchseuchung der Bevölkerung reflektiert,

2. die Virusisolierungen die Anteile der Influenzatypen und -subtypen an der Morbidität wiedergeben.

Im Mittelpunkt der Betrachtungen stehen hier nicht Inzidenzen und absolute Werte, sondern die Veränderung der Morbidität und somit auch Durchseuchung von Jahr zu Jahr, also die Rhythmik der Influenzaaktivität.

Mit den Sentinel- und virologischen $\mathrm{Da}$ ten soll ein Modell entwickelt werden, anhand dessen Zusammenhänge zwischen den Influenza-assoziierten Morbiditäten vorangegangener Jahre mit der im Folgejahr auftretenden Morbidität untersucht werden können.

Die Zuordnung der gesamten Influenza-bedingten Morbidität zu den einzelnen Typen und Subtypen kann anhand der Anteile der Isolierungen der verschiedenen Influenzatypen und -subtypen erfolgen. Dies ermöglicht eine getrennte Betrachtung der Zusammenhänge zwischen Morbidität in den vergangenen Jahren und beobachteter Morbidität im Folgejahr für jeden Influenzatyp und -subtyp.

Um die komplizierenden Einflüsse der Drift gering zu halten und so ein möglichst einfaches Modell zu erhalten, konzentrieren sich diese ersten Untersuchungen auf Influenza $B$, die eine geringere Drift aufweist als Influenza A/H3N2.

Anhand der Influenza-Survcillancedaten von $1988 / 89$ bis $1997 / 98$ wurde für 
Tabelle 1: Anzahl der Isolierungen der Influenzatypen und -subtypen und deren Anteil an den gesamten Isolierungen von $1988 / 89$ bis $1997 / 98$

\begin{tabular}{rrrrrrrr}
\hline & $\begin{array}{c}\text { gesamt } \\
=100 \%\end{array}$ & \multicolumn{2}{c}{$\mathrm{A}(\mathrm{H} 3 \mathrm{~N} 2)$} & \multicolumn{2}{c}{$\mathrm{A}(\mathrm{H} 1 \mathrm{~N} 1)$} & \multicolumn{3}{c}{$\mathrm{B}$} \\
Jahr & Isolate & Isolate & $\%$ & Isolate & $\%$ & Isolate & $\%$ \\
\hline $88 / 89$ & 68 & 13 & 19 & 52 & 76 & 3 & 4 \\
$89 / 90$ & 247 & 203 & 82 & 0 & 0 & 44 & 18 \\
$90 / 91$ & 87 & 0 & 0 & 13 & 15 & 74 & 85 \\
$91 / 92$ & 86 & 59 & 69 & 27 & 31 & 0 & 0 \\
$92 / 93$ & 73 & 12 & 16 & 0 & 0 & 61 & 84 \\
$93 / 94$ & 11 & 11 & 100 & 0 & 0 & 0 & 0 \\
$94 / 95$ & 112 & 21 & 19 & 2 & 2 & 89 & 79 \\
$95 / 96$ & 365 & 201 & 55 & 152 & 42 & 12 & 3 \\
$96 / 97$ & 423 & 167 & 39 & 24 & 6 & 232 & 55 \\
$97 / 98$ & 723 & 669 & 96 & 51 & 7 & 3 & 1 \\
\hline
\end{tabular}

Influenza B ein neues Modell für das Wechselspiel von Populationsimmunität und Influenzaaktivität entwickelt. Mit Hilfe retrospektiver Betrachtungen der in den einzelnen Jahren von dem Subtyp B verursachten Morbidität (Durchseuchung) und somit Immunität und deren Vergleich mit der im Folgejahr gemessenen von diesem Subtyp verursachten Morbidität soll eine erste Bewertung dieses einfachen Modells vorgenommen werden.

\section{Material und Methoden}

\section{Virusisolate}

Seit Bestehen der Arbeitsgemeinschaft Influenza (AGI) werden im nationalen Referenzzentrum für Influenza Abstriche aus etwa $5 \%$ der Sentinel-Praxen virologisch untersucht. Die Anzahl der untersuchten Proben konnte so mit dem Ausbau des Sentinels erheblich gesteigert werden. Darüber hinaus werden auch Proben aus Krankenhäusern, weiteren Arztpraxen und virologischen $\mathrm{La}$ bors in die Untersuchungen einbezogen. Der überwiegende Anteil der Proben stammt aus primärversorgenden Arztpraxen, die an den Morbiditätsbeobachtungen beteiligt sind.

In den Jahren vor 1992/93 war die Zahl der untersuchten Proben geringer und stammte aus Krankenhäusern, virologischen Labors sowie einigen primärversorgenden Arztpraxen in Niedersachsen.

Die Zahl der untersuchten Proben war überwiegend von der Influenzaaktivität abhängig und konstanter als nach 1992/93.
Tabelle 1 gibt die Anzahl der Isolierungen der einzelnen Influenzatypen und -subtypen in Deutschland von $1988 / 89$ bis $1997 / 98$ wieder und deren Anteil an den Isolierungen insgesamt.

\section{Morbidität}

Als Datenmaterial stehen die Beobachtungen der AGI, die das Winterhalbjahr von der 40 . KW bis zur 17 . KW des folgenden Jahres umfassen, zur Verfügung. Diese beinhalten die Beobachtungsperioden $1992 / 93$ bis $1997 / 98$. In mehr als 600 Arztpraxen bundesweit werden alle akuten Atemwegsinfektionen registriert. Als Bezugsgröße, die mit der beobachteten Bevölkerung korreliert, wird die Anzahl der gesamten Praxiskontakte genutzt. Die Beobachtungen basieren auf insgesamt mehr als 100000 wöchentlichen Patientenkontakten.

Um die Influenza-assoziierte Morbidität abschätzen zu können, wurden anhand der vergangenen Beobachtungsperioden wöchentliche Erwartungswerte der ARE-Morbidität kalkuliert (Basislinie) $[3,4]$. Dies erlaubt ein Abschätzen der zusätzlichen, z. B. durch eine erhöhte Influenzaaktivität verursachten Morbidität.

Für jede Beobachtungsperiode wurde die Influenza-assoziierte Morbidität errechnet als Summe der über den Erwartungswert hinausgehenden Beobachtungswerte über alle Wochen, in denen auch die Virusisolierungen mehr als eine sporadische Influenzaverbreitung anzeigen (siehe Beispiel 1).

Als Grundlage wurde hier der Praxisindex genutzt, da mir diesem Index eine präzisere Bewertung der Influenzaaktivität in den betrachteten Beobachtungsperioden möglich war [5].

$\mathrm{Da}$ es sich um eine relative Betrachtung (über die Zeit aufsummierte Stärke der Überschreitung im Vergleich zu den anderen Jahren) handelt, können aber auch andere Parameter mit den jeweiligen Vor- und Nachteilen verwendet werden (ARE je Praxis, EISS Index [6], ARE je 100 Praxiskontakte etc.).

Die Werte für die Durchseuchung in den Beobachtungsperioden vor 1992/93 wurden nur aus der Anzahl der Virusisolierungen abgeschätzt. Dazu wurden die zum Gipfelpunkt beobachteten Morbiditäten 1989/90 mit H3N2 den 1995/96 beobachteten etwa gleichgesetzt. Aufgrund der geringeren $\mathrm{Zahl}$ an Wochen mit erhöhter Influenzaaktivität ist der Gesamtwert 1989/90 etwas geringer als 1995/96.

Vergleiche der Mortalitätszahlen sowie der Bewertungen der Influenzaaktivität aus den Nachbarländern wurden dabei berücksichtigt.

Daraus resultierten folgende Werte der gesamten Influenza-assoziierten Morbidität während einer Beobachtungsperiode (Durchseuchungsindex gesamt). Als Beispiel für die Berechnung sei die Beobachtungsperiode 1994/95 dargestelit.

\section{Beispiel 1}

Berechnung des Durchseuchungsindex für 1994/95:

Einbezogen wurden die Wochen 7 bis 141995 , in denen eine nennenswerte Influenzaverbreitung nachgewiesen wurde. Dabei waren die Erwartungswerte in der 7 . KW um $6 \%$ in der 8 . KW um $15 \%$, in der $9 . \mathrm{KW}$ um $30 \%$, in der 10. $\mathrm{KW}$ um $48 \%$, in der 11 . KW um $54 \%$, in der 12 . KW um $59 \%$, in der 13. KW um $51 \%$ und in der 14 . KW um $36 \%$ überschritten. Die Summe stellt den Durchseuchungsindex dar und ergibt 299.

Die Verteliung auf die einzelnen Influenzatypen und -subtypen erfolgt anhand der jeweiligen Anteile der Isolierungen (Tab.1). Für das Beobachtungsjahr 1994/95 ergibt sich für Influenza B mit einem Isolierungsanteil von $79 \%$ ein Anteil von 233 an dem Durchseuchungsindex.

Die Werte der Durchseuchungsindizes für die einzelnen Influenzatypen und -subtypen für die einzelnen Beob- 
Tabelle 2: In den einzelnen Beobachtungsperioden aus den Morbiditätsdaten kalkulierte Durchseuchungsindizes gesamt und deren Verteilung auf die einzelnen Influenzatypen und -subtypen

\begin{tabular}{lllll}
\hline Jahr & $\begin{array}{l}\text { Durchseuchungs- } \\
\text { index gesamt }\end{array}$ & $\begin{array}{l}\text { für } \\
\mathrm{A}(\mathrm{H} 3 \mathrm{~N} 2)\end{array}$ & $\begin{array}{l}\text { für } \\
\mathrm{A}(\mathrm{H} 1 \mathrm{~N} 1)\end{array}$ & $\begin{array}{l}\text { für } \\
\mathrm{B}\end{array}$ \\
\hline $88 / 89$ & 230 & 44 & 185 & 9 \\
$89 / 90$ & 700 & 574 & 0 & 126 \\
$90 / 91$ & 300 & 0 & 45 & 255 \\
$91 / 92$ & 260 & 179 & 81 & 0 \\
$92 / 93$ & 552 & 88 & 0 & 464 \\
$93 / 94$ & 260 & 260 & 0 & 0 \\
$94 / 95$ & 299 & 57 & 6 & 236 \\
$95 / 96$ & 881 & 623 & 233 & 25 \\
$96 / 97$ & 464 & 181 & 28 & 255 \\
$97 / 98$ & 262 & 244 & 14 & 3 \\
\hline
\end{tabular}

achtungsperioden sind in Tabelle $2 \mathrm{dar}$ gestellt.

Die Bedeutung der Durchseuchung in den vergangenen Jahren für die Bevölkerungsimmunität nimmt mit der Zeit ab. Dabei können ein Absinken der Immunität nach einer Infektion durch sinkende Antikörpertiter und die driftbedingten Veränderungen der Oberflächenantigene des Virus als Ursachen vermutet werden.

Die für die Bevölkerungsimmunität entscheidende Abnahme der Bedeutung der Durchseuchung der vorangegangenen Jahre ist nicht bekannt. Dieser un-

Tabelle 3: Durchseuchungsindex für Influenza $B$ in den einzelnen Beobachtungsperioden und der entsprechende aus den fünf vorherigen Durchseuchungsindizes abgeschätzte Immunitätsindex

\begin{tabular}{lcl}
\hline Jahr & $\begin{array}{l}\text { Durch- } \\
\text { seuchungs- } \\
\text { index für } \\
\text { Influenza B }\end{array}$ & $\begin{array}{l}\text { Immunitäts- } \\
\text { index } \\
\text { für } \\
\text { Influenza B }\end{array}$ \\
\hline $88 / 89$ & 9 & \\
$89 / 90$ & 126 & \\
$90 / 91$ & 255 & \\
$91 / 92$ & 0 & $171^{*}$ \\
$92 / 93$ & 464 & 528 \\
$93 / 94$ & 0 & 320 \\
$94 / 95$ & 236 & 441 \\
$95 / 96$ & 25 & 259 \\
$96 / 97$ & 255 & 438 \\
$97 / 98$ & 3 & 198 \\
$98 / 99$ & & \\
\hline
\end{tabular}

* (nur vier Jahre) bekannte Faktor soll empirisch anhand des Modells bestimmt werden.

Hierfür wurde aus den Durchseuchungsindizes der vorangegangenen fünf Beobachtungsperioden ein Immunitätsindex (relative Immunität gegen Influenza B) mit einer einheitlichen Formel unter Einbeziehung des unbekannten Faktors für die abnehmende Relevanz ermittelt. Der Faktor wurde mit linearen und verschiedenen exponentiellen Verläufen für die Wichtung der Durchseuchung der vergangenen Beobachtungsperioden mit dem zeitlichen Abstand zum betrachteten Winterhalbjahr getestet.

Der Korrelationskoeffizient zwischen dem Immunitätsindex (aus den zurückliegenden fünf Beobachtungsperioden) und dem tatsächlich beobachteten Durchseuchungsindex im folgenden Jahr diente als Bewertungsmaßstab.

\section{Ergebnisse}

Ein Zusammenhang des Immunitätsindex mit der Durchseuchung in der betrachteten Beobachtungsperiode konnte für Influenza $B$ hergestellt werden (Tab. 3).

Bei einer Abnahme der Bedeutung der Durchseuchung für die Immunität mit jedem Jahr um 1/Jahresabstand konnte eine enge Korrelation erreicht werden.

Die Berechnung des Immunitätsindex für alle Beobachtungsperioden nach der im folgenden aufgeführten Formel zeigte zufriedenstellende Ergebnisse und einen hohen Korrelationskoeffizienten von $-0,96$.

$$
\sum_{i=1}^{5} D_{i} * \frac{1}{i}
$$

$\mathrm{D}=$ Durchseuchungsindex

$\mathrm{i}=$ Abstand von dem zu prüfenden Beobachtungsjahr in Jahren

\section{Beispiel 2}

Berechnung des Immunitätsindex 1997/98:

(Durchseuchungsindex $=\mathrm{D}$ ) Immunitätsindex $97 / 98=[\mathrm{D} 96 / 97+\mathrm{D} 95 / 96$ $* 1 / 2+$ D $94 / 95 * 1 / 3+$ D $93 / 94 * 1 / 4+D$ $92 / 93 * 1 / 5]$

Der enge Zusammenhang zwischen Immunitätsindex und dem beobachteten Durchseuchungsindex in den untersuchten Beobachtungsperioden wird auch durch die Darstellung des Korrelationsdiagrammes der Wertepaare 1992/93 bis 1997/98 deutlich (Abb.1).

Für eine lineare Regression ergibt sich die Formel $Y=-0,684 * X+471,6$.

Daraus kann man für jeden Immunitätsindex den entsprechenden Erwartungswert der Durchseuchung errechnen.

Stellt man die beobachteten Durchseuchungsindizes für Influenza $\mathrm{B}$ den jeweiligen Erwartungswerten gegenüber, erhält man Tabelle 4.

Die Abweichung der beobachteten Durchseuchung von der nach diesem Modell erwarteten Durchseuchung ist in allen einbezogenen Beobachtungsperioden gering. Für die kommende Beobachtungsperiode 1998/99 kann nach diesem Modell eine stärkere Influenza-BDurchseuchung (mehr als 96/97 und weniger als im März 1993) erwartet wer$\operatorname{den}[7]$.

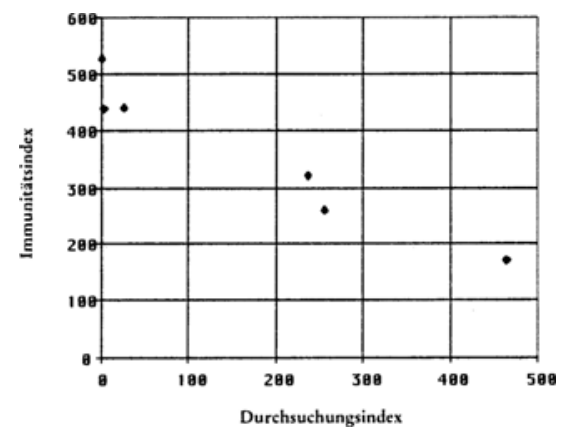

Abbildung 1: Immunitäts- und Durchseuchungsindex gegenüber Influenza $B$ in der Bundesrepublik Deutschland von der Beobachungsperiode $1992 / 93$ bis $1997 / 98$. 
Tabelle 4: Immunitätsindex, beobachtete Durchseuchung und erwartete Durchseuchung mit Influenza B von 1992/93 bis $1998 / 99$

\begin{tabular}{llll}
\hline Jahr & $\begin{array}{l}\text { Immunitätsindex } \\
\text { für Influenaz B }\end{array}$ & $\begin{array}{l}\text { Beobachteter } \\
\text { Durchseuchungsindex } \\
\text { für Influenza B }\end{array}$ & $\begin{array}{l}\text { Erwartungswert für den } \\
\text { Durchseuchungsindex } \\
\text { für Influenza B }\end{array}$ \\
\hline $92 / 93$ & $171^{*}$ & 464 & 439 \\
$93 / 94$ & 528 & 0 & $(-83) 0$ \\
$94 / 95$ & 320 & 236 & 221 \\
$95 / 96$ & 441 & 25 & 44 \\
$96 / 97$ & 259 & 255 & 310 \\
$97 / 98$ & 438 & 3 & 48 \\
$98 / 99$ & 198 & & 399 \\
\hline
\end{tabular}

* (nur vier Jahre, s. Tab. 3)

\section{Diskussion}

Die enge Korrelation der Morbidität in den vergangenen Jahren mit der Morbidität im kommenden Jahr über sechs Jahre unter Einbeziehung von Daten aus zehn Beobachtungsjahren ist nur mit einer geringen Wahrscheinlichkeit zufallsbedingt.

Eine mögliche Ursache für eine gewisse Ungenauigkeit der Ausgangsdaten stellt die während der Beobachtungsperioden von 1988/89 bis 1991/92 nur aus den virologischen Daten abgeschätzte Durchseuchung dar. Aus Holland und der Schweiz liegen für die gesamte Zeitspanne sentinelgestützte epidemiologische und virologische Beobachtungsdaten vor. Diese lassen die für die Bundesrepublik Deutschland abgeschätzten Daten in dieser Zeitspanne plausibel erscheinen. Insgesamt konnte für Influenza $B$ aus den Daten aus Holland und der Schweiz von $1988 / 89$ bis $1997 / 98$ mit dem gleichen Verfahren ein ähnlich hoher Korrelationskoeffizient errechnet werden (hier nicht dargestellt). Daher scheinen gravierende Ungenauigkeiten aus den anhand der virologischen Daten abgeschätzten Durchseuchung von $1988 / 89$ bis 1991/92 nicht gegeben.

Weitere Verbesserungen der Genauigkeit der Ausgangsdaten sind durch eine Berücksichtigung möglicher regionaler Unterschiede der Influenzaaktivität und der entsprechenden Bevölkerungsverteilung gegeben. Möglicherweise ist auch eine wöchentliche Zuordnung zu den Influenzatypen und -subtypen mit anschließender Addition der Wochenwerte etwas präziser als die hier verwen- dete Verteilung der gesamten Durchseuchung.

Nachteilig für die endgültige Bewertung des Modells ist die begrenzte Anzahl der Beobachtungsjahre. Daran ändern auch die möglichen Vergleiche mit den Daten aus Holland und der Schweiz nichts, da die Muster der InfluenzaB-Aktivität in diesen Ländern in der betrachteten Zeitspanne ähnlich war. Für die Zukunft sind Vergleiche mit weiteren Ländern, die Daten ähnlicher Qualität über diese Zeitspanne bereitstellen können, geplant. Dabei sind besonders Länder mit abweichender jährlicher Verteilung der Influenza-BAktivität, also abweichender Rhythmik von den bisher einbezogenen Ländern, interessant.

Zum jetzigen Zeitpunkt sind valide Prognosen über eine zu erwartende Influenza-B-Aktivität mit Hilfe des Modells noch nicht möglich. Dafür ist die Zahl der untersuchten Beobachtungsjahre noch zu gering. Die virologischen $\mathrm{Un}_{\mathrm{n}}$ tersuchungen legen für den Zeitraum von $1988 / 89$ bis $1997 / 98$ eine geringe Drift von Influenza B nahe. Es ist daher nicht klar, wie sich das Modell bei einer stärkeren Drift verhält.

Das Modell erhärtet jedoch die Annahme, daß ein Zusammenhang zwischen beobachteter Morbidität in einer Beobachtungsperiode (als Maßstab für die Durchseuchung) und der Immunität als Resultat aus der Durchseuchung in vergangenen Jahren zumindest für Influenza B besteht. Weitere Arbeiten mit diesem Modell und verfeinerten Modellen für Influenza A (Drift) lassen even- tuell auch die Relevanz einer Drift für die Immunität erkennen.

Dieser erste Versuch, die Erkenntnisse aus Virologie und Epidemiologie der Influenza zu verknüpfen, läßt eine Reihe weiterer gemeinsamer Untersuchungsschwerpunkte erkennen. Die Bewertung der Drift für die Populationsimmunität, die Bedeutung und Anteile von leichter Erkrankten für die Verbreitung der Influenza oder die Mechanismen bei der Etablierung einer neuen Variante seien hier beispielhaft genannt.

\section{Danksagung:}

Wir möchten uns besonders bei den Ärztinnen und Ärzten sowie dem Personal in den Sentinel-Praxen bedanken, die mit ihrer freiwilligen und unentgeltlichen Mitarbeit die AGI und deren epidemiologische Beobachtungen erst ermöglichen. Unser herzlicher Dank gilt ebenfalls Dr. Werner Wunderli, Dr. Jann Cloetta, Dr. Jan de Jong und Dr. Aad Bartelds für die Bereitstellung der Daten aus den Nachbarländern sowie den Mitarbeitern der vielen Institutionen, die mit der AGI zusammenarbeiten und sie mit Rat und Tat unterstützen.

\section{Literatur:}

[1] Szecsenyi, J., Uphoff, H., and Brede, H. D.: Influenza surveillance: experience from establishing a sentinel surveillance system in Germany. Jour, of Epid. and Community health 49 (1995) 9-13.

[2] Uphoff, H.: Influenza-Surveillance in der Bundesrepublik Deutschland. J. Lab. Med. 20, 6 (1996) $375-378$

[3] Uphoff, H., und Szecsenyi, J.: Influenza-Monitoring mit dem bundesweiten Sentinel-System der Arbeitsgemeinschaft Influenza (AGI). InfFo 1/96 6-11.

[4] Lange, W., Rasch, G., and Uphoff, H.: Surveillance of influenza in Germany: results of a sentinel and laboratory reporting system. Options for the Control of Influenza III 1996; Elsevier Science B. V. 65-70.

[5] Uphoff, H.: Der »Praxisindex« als eine Größe für regionale Betrachrungen der Influenzaaktivitär. Robert Koch-Institut (Hrsg.): InfFo III/IV (1998).

[6] Uphoff, H.: European Influenza Surveillance Scheme (EISS): Eine vereinfachte Darstellung nationaler Influenza-Surveillance-Daten. Robert Koch-Institut (Hrsg.): InfFo III/IV (1998).

[7] Uphoff, H., Heckler, R., Rasch, G., Schweiger, B., und Lange, W.: Influenza-Aktivität im Winter 1996/97. Bundesgesundhbl. 40, 10 (1997) $385-392$

Anschrift der Verfasser

Dr. Helmut Uphoff, Arbeitsgemeinschaft Influenza (AGI), Schumarkt 4, 35037 Marburg/Lahn; Dr. Rolf Heckler, Nationales Referenzzentrum für Influenza Hannover, Roesbeckstr. 4, 30449 Hannover; Dr. Brunhilde Schweiger, Nationales Referenzzentrum für Influenza, Robert KochInstitut, Postfach 6502 80, 13302 Berlin 